\title{
Role of miRNA-21 in the diagnosis and prediction of treatment efficacy of primary central nervous system lymphoma
}

\author{
KUNPENG YANG, SHUYE WANG, YAFENG CHENG, YAOYAO TIAN and JINXIAO HOU \\ Department of Hematology, The First Affiliated Hospital of Harbin Medical University, \\ Harbin, Heilongjiang 150001, P.R. China
}

Received March 1, 2018; Accepted December 13, 2018

DOI: $10.3892 /$ ol.2019.9941

\begin{abstract}
Primary central nervous system lymphoma (PCNSL) has a poor prognosis and requires early diagnosis and treatment. The aim of the present study was to investigate the difference between microRNA-21 (miRNA-21) expression in the plasma and cerebrospinal fluid (CSF) of patients with PCNSL, and to discuss the importance of miRNA-21 in its diagnostic and therapeutic evaluation. The research subjects were confirmed as patients with PCNSL with histopathological lesions at The First Affiliated Hospital of Harbin Medical University (Harbin, China) between December 2011 and 2017. Comparisons were drawn between the PCNSL, glioblastoma and the healthy control groups. CSF and plasma specimens were obtained from patients with PCNSL prior to chemotherapy, and CSF specimens were also obtained following chemotherapy. Plasma specimens were taken from patients with glioblastoma and the healthy control group. Using reverse transcription-quantitative polymerase chain reaction analysis, it was revealed that plasma miRNA-21 expression level had a notable diagnostic value in distinguishing PCNSL from glioblastoma, another common neurological tumor. Moreover, miRNA-21 expression levels in the plasma correlated positively with those in the CSF. Therefore, miRNA-21 in the plasma may be used as a novel diagnostic biomarker to distinguish patients with PCNSL from those with glioblastoma, whereas miRNA-21 in the CSF may have potential as a predictor of chemotherapeutic effect in PCNSL.
\end{abstract}

Correspondence to: Professor Shuye Wang, Department of Hematology, The First Affiliated Hospital of Harbin Medical University, 23 Youzheng Street, Nangang, Harbin, Heilongjiang 150001, P.R. China

E-mail: 16745179@qq.com

Key words: primary central nervous system lymphoma, microRNA-21, diagnostic marker, efficacy evaluation marker, glioblastoma

\section{Introduction}

Primary central nervous system lymphoma (PCNSL) is an aggressive non-Hodgkin's lymphoma that is confined to the central nervous system (CNS). It is a rare tumor that commonly exhibits the morphological and immunophenotypical features of diffuse large B-cell lymphoma (DLBCL). PCNSL frequently occurs in the brain, eyes, pia mater and spinal cord, accounting for $~ 3 \%$ of CNS tumors (1). The disease has a poor prognosis, with a life expectancy without treatment of 3-5 months.

The only known risk factor for PCNSL is immunodeficiency, including as a result of acquired immunodeficiency syndrome (AIDS), transplant and immunosuppressive therapy, inherited immunodeficiency and other acquired immune deficiencies (2). Human Epstein-Barr virus infection may be important in the etiology of PCNSL in immunocompromised patients (2). Other etiological possibilities include in situ malignant lymphocytes, clonal hyperplasia in the CNS, dysplasia of lymphocytes the CNS and the malignant transformation of lymphocytes following CNS infection (3). Studies have reported that PCNSL occurs as a result of genetic alterations, and the occurrence of lymphoma is heavily associated with microRNAs (miRNAs) (4-6). Zheng et al (7) demonstrated that the miRNA expression of primary central nervous system (PCNS)-DLBCL differed from that of germinal center (GC)-DLBCL and non-GC-DLBCL. miRNA-21, located on chromosome $17 \mathrm{q} 23.2$, presents in a number of solid and non-solid tumors. The expression of miRNA-21 is increased in DLBCL, follicular lymphoma, and transformational DLBCL lymphoid tissue compared with healthy individuals (8). The present study aimed to determine the expression and diagnostic value of miRNA-21 in PCNSL. The value of miRNA-21 in distinguishing glioblastoma, another common tumor in CNS, was also assessed in addition to predicting the therapeutic effect of chemotherapy in PCNSL, which has not been reported previously.

\section{Patients and methods}

Clinical information. A total of 25 patients with PCNSL from the First Affiliated Hospital of Harbin Medical University (Harbin, China) between December 2011 and December 2017 were enrolled in the present study (male, 13; female, 12; median age, 56.6 years; range, 36-69 years). The inclusion 
criteria were as follows: i) Patients with or without an impaired performance of the CNS as the primary symptoms (headache, dizziness, nausea, vomiting and other elevated intracranial pressure symptoms; epilepsy, aphasia, visual impairment, limb weakness, unsteady gait and other neurological symptoms). Lymphoma lesions were localized in the CNS and were all confirmed by histopathological examination; ii) all patients received bone marrow biopsy and an ultrasound to rule out systemic lymphoma; iii) following detailed physical and auxiliary examinations, no systemic lymphoid hematopoietic tissue or other system involvement was confirmed; iv) human immunodeficiency virus (HIV) antibody test was negative. The exclusion criteria were as follows: i) cancer; ii) pregnancy; iii) severe infection; iv) severe cardiovascular or cerebrovascular diseases; v) heart, liver or kidney dysfunction; and vi) autoimmune disease. The control groups were 25 patients with glioblastoma $($ male $=11$, female $=14$; median age, 57 years; range, 44-67 years) and 25 healthy volunteers (male $=13$, female $=12$; median age: 57.2 years; range: 43-68 years). The inclusion criteria were as follows: i) Patients diagnosed with glioblastoma or volunteers without CNS diseases; and ii) HIV antibody test was negative. The exclusion criteria were the same as for the PCNSL group.

The Harbin Medical University Ethics Committee (Harbin, China) approved the present study, and all blood samples or cerebrospinal fluid (CSF) samples were used with the written informed consent of the patients.

Imaging and clinical evaluations. Cranial computed tomography or magnetic resonance imaging was used to indicate the site of intracranial invasion. The Karnofsky performance status (KPS) score (9) was used to assess the performance of patients with PCNSL in daily activities.

Laboratory examinations. Lactate dehydrogenase (LDH) in the serum and CSF were examined prior to treatment at the clinical laboratory of The First Affiliated Hospital of Harbin Medical University. $L D H$ was detected using the Beckman LDH kit (Beckman Coulter, Inc., Brea, CA, USA). Protein and sugar contents in CSF were measured with dry chemistry method (VITROS; Ortho-Clinical Diagnostics, Inc., Rochester, NY, USA). Leukocytes were counted using a light microscope (magnification, $\mathrm{x} 40$ ).

Plasma and CSF samples. Peripheral blood samples (3 ml) from patients with PCNSL, with glioblastoma, and healthy volunteers were collected into EDTA anticoagulant tubes prior to chemotherapy, and processed within $4 \mathrm{~h}$ of collection. All patients with PCNSL received high dose methotrexate (MTX)-based chemotherapy following admission to hospital. CSF was taken during lumbar intrathecal chemotherapeutic injection. The CSF was collected into sterile collection tubes prior to, following one cycle, and following three cycles of chemotherapy, and was processed within $4 \mathrm{~h}$ of collection. Plasma and CSF were isolated at $4^{\circ} \mathrm{C}$ under centrifugation for $10 \mathrm{~min}(1,500 \mathrm{x} \mathrm{g})$. The supernatant was transferred to a clean tube and the procedure was repeated prior to storage at $-80^{\circ} \mathrm{C}$.

RNA extraction and reverse transcription-quantitative polymerase chain reaction ( $R T-q P C R)$. Total RNA was extracted from patient samples using a TRIzol ${ }^{\circledR}$ extraction kit (Invitrogen; Thermo Fisher Scientific, Inc., Waltham, MA, USA), according to the manufacturer's protocol. Optical density (OD) and RNA concentration were determined using a UV spectrophotometer at 260 and $280 \mathrm{~nm}$. Zero-point adjustment prior to measurement was conducted with diethylpyrocarbonate-treated water. Each sample was tested twice, and an OD260/OD280 ratio of 1.8-2.0 was deemed acceptable.

To detect miRNA-21, RT-qPCR was performed, according to the manufacturer's protocol. Briefly, $20 \mu \mathrm{l}$ of each sample (100 ng total RNA) was used in the RT reactions $\left(42^{\circ} \mathrm{C}\right.$ for $50 \mathrm{~min}, 95^{\circ} \mathrm{C}$ for $5 \mathrm{~min}$, followed by $4^{\circ} \mathrm{C}$ ). qPCR was conducted using the following conditions: $95^{\circ} \mathrm{C}$ for $5 \mathrm{~min}, 40$ cycles of $30 \mathrm{sec}$ at $95^{\circ} \mathrm{C}$, and $40 \mathrm{sec}$ at $72^{\circ} \mathrm{C}$. The miRNA expression levels were calculated as the quantification cycle $\left(2^{-\Delta \Delta \mathrm{Cq}}\right)$ of miRNA-21 (10). $\beta$-actin (forward, 5'-GGCACCCAGCACAAT GAAG-3', and reverse, 5'-CGTCATACTCCTGCTTGCTG-3') was used as the internal control. RT reactions and qPCR were performed using a SuperScript VILO Synthesis kit (Thermo Fisher Scientific, Inc.) and the SsoAdvanced SYBR ${ }^{\circledR}$ Green Supermix (Bio-Rad Laboratories, Inc., Hercules, CA, USA). The primers used were as follows: miRNA-21 forward, 5'-GTG CAGGGTCCGAGGT-3', and reverse, 5'-GCCGCTAGCTTA TCAGACTGATGT-3'.

Statistical analysis. Each experiment was repeated two more times and the mean value was calculated. Values for miRNA-21 in the plasma and CSF are displayed as the mean \pm standard deviation. Data between the PCNSL and the control groups were compared using analysis of variance with Student-Newman-Keuls post hoc test. Correlations between miRNA-21 expression levels in the plasma and those in the CSF were assessed using the Pearson's correlation coefficient. Receiver operating characteristic (ROC) analysis was used to determine diagnostic values. The Wilcoxon test was used to test the value changes in CSF miRNA-21 prior to and following chemotherapy, in patients who responded to chemotherapy and those who did not. Spearman's rank correlation coefficient was used to test the reliability and validity of miRNA-21 in predicting therapeutic effect. Statistical analyses were conducted using SPSS 19.0 (IBM Corp., Armonk, NY, USA). P<0.05 was considered to indicate a statistically significant difference.

\section{Results}

Clinical features of patients with PCNSL. Following donation, 25 plasma samples and 75 CSF samples for each time point were analyzed (prior to, following one cycle, and following three cycles of chemotherapy) from 13 male and 12 female patients with PCNSL, and 50 plasma samples from the control groups (25 patients with glioblastoma and 25 healthy volunteers). There was no significant difference in sex or age between different groups $(\mathrm{P}>0.05)$. The pathological types of the patients with PCNSL were all B-cell type lymphoma, in which DLBCL accounted for 23 cases, and the other 2 cases were unclassified B-cell type lymphoma. Headache, nausea, vomiting, and other symptoms of intracranial hypertension were the most common symptoms. Visual impairment, limb weakness, numbness, paraplegia, and other physical disabilities were also observed. 
Table I. Cerebrospinal fluid analyses of patients with PCNSL.

\begin{tabular}{lccr}
\hline Cases of PCNSL & Leukocyte count, n (\%) & Protein content, n (\%) & Sugar content, n (\%) \\
\hline Within normal limits & $15(60.0)$ & $8(32.0)$ & $18(72.0)$ \\
Beyond normal limits & $10(40.0)$ & $17(68.0)$ & $7(28.0)$ \\
\hline
\end{tabular}

PCNSL, primary central nervous system lymphoma; CSF, cerebrospinal fluid.

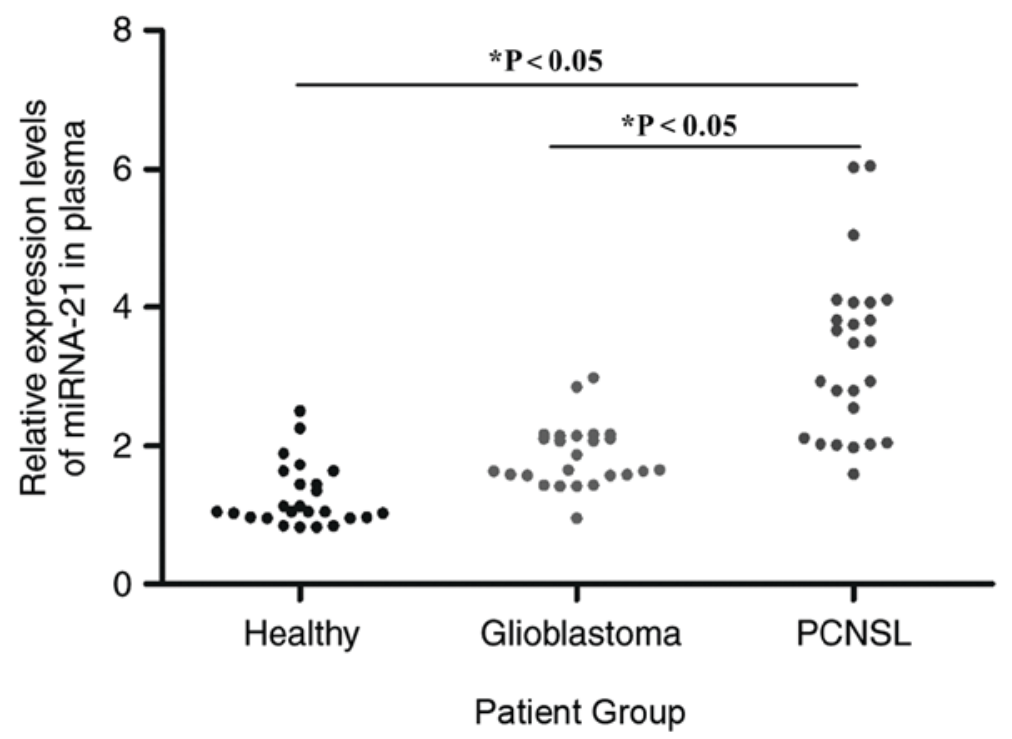

Figure 1. miRNA-21 expression levels in the PCNSL, glioblastoma and healthy control groups. The relative expression level of plasma miRNA-21 was higher in the PCNSL group compared with the other two groups (" $\mathrm{P}<0.05)$. There was a minor overlap in the distribution of the PCNSL and the glioblastoma groups, while there was a greater degree of overlap between the glioblastoma and the healthy control groups. miRNA, microRNA; PCNSL, primary central nervous system lymphoma.

Imaging and clinical evaluations. All patients with PCNSL underwent imaging examinations by cranial computed tomography or magnetic resonance imaging. Imaging data revealed that the frontal lobe was most frequently affected (data not shown).

The KPS score was used to assess the performance of the 25 patients with PCNSL in daily activities. The KPS score was $\geq 80$ in 15 cases, and $<80$ in 10 cases (the scores of 3 cases were $<60)$. The majority of the patients remained independent with relatively mild symptoms (data not shown).

Laboratory examination. Of the 25 patients with PCNSL, LDH in the serum of 6 cases (24\%) was aberrantly elevated, while it was normal in 19 cases (76\%). CSF was also tested. The normal group was defined by leukocyte number, protein and sugar content within the normal limits. Samples outside of these limits were assigned to the abnormal group. CSF examinations revealed that the majority of patients with PCNSL had a normal leukocyte count and sugar content, but abnormal protein content. Patient number and percentage in normal and abnormal groups are displayed in Table I.

Expression and diagnostic value of plasma miRNA-21 in $P C N S L$. The relative expression levels of miRNA-21 in the plasma of the PCNSL, glioblastoma, and healthy groups were assessed using RT-qPCR prior to chemotherapy. The
miRNA-21 expression levels in the plasma of the PCNSL group were significantly higher when compared with that in the other groups. In addition, miRNA-21 expression in the plasma of the glioblastoma group was also significantly higher compared with that in the healthy group $(\mathrm{P}<0.05)$. The relative expression levels of miRNA-21 were higher in the PCNSL group and there was a slight overlap in the distribution of miRNA-21 in the PCNSL group and the glioblastoma group, while there was a greater overlap between the glioblastoma group and the healthy group (Fig. 1). The ROC curve illustrates that miRNA-21 in the plasma had a high specificity for distinguishing PCNSL from the healthy control group [area under the curve (AUC), 0.971; 95\% confidence interval (CI), 0.933-1.000] (Fig. 2A) and glioblastoma group (AUC, 0.862; 95\% CI, 0.763-0.962) (Fig. 2B).

Correlation of miRNA-21 expression in CSF and plasma in PCNSL. By comparing the expression levels of miRNA-21 in plasma and CSF prior to chemotherapy in patients with PCNSL, the correlation analysis revealed a significantly positive correlation (Pearson's correlation coefficient: $\mathrm{r}^{2}=0.708$, $\mathrm{P}<0.001$ ) (Fig. 3).

miRNA-21 expression levels in the CSF of patients with PCNSL prior to and following chemotherapy. Patients admitted to the hospital were predominantly treated with 

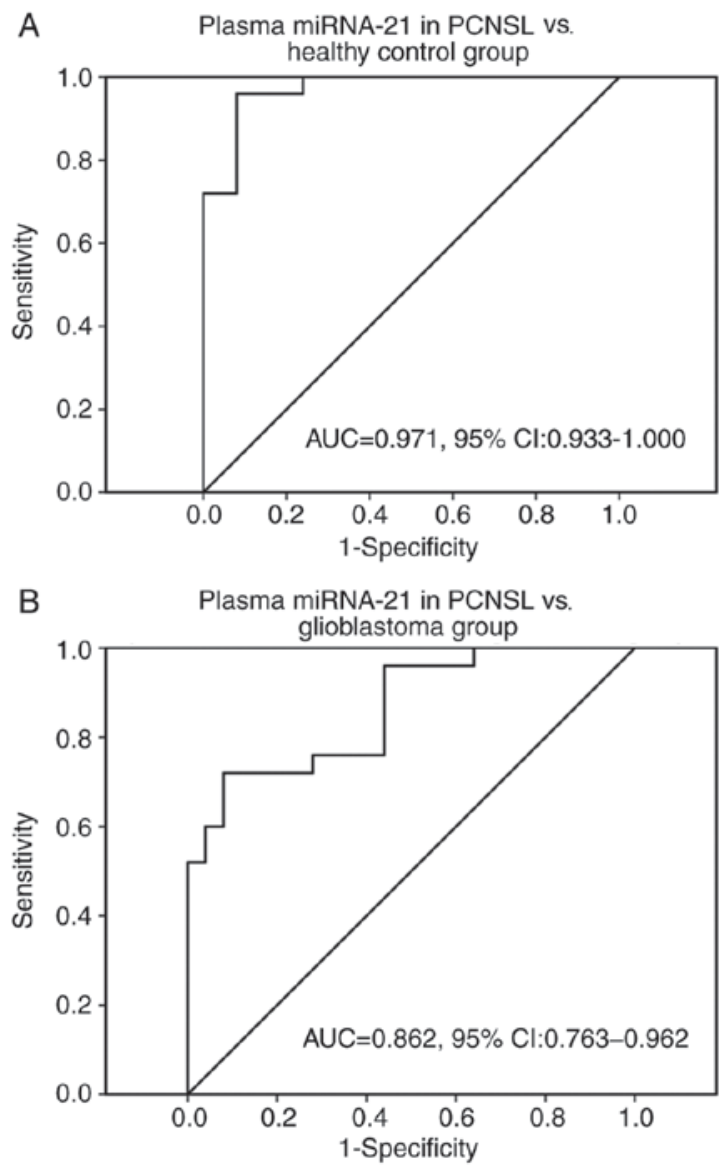

Figure 2. Diagnostic value of plasma miRNA-21 in PCNSL. (A) ROC curve for plasma miRNA-21 for patients with PCNSL compared with the healthy control group. (B) ROC curve for plasma miRNA-21 for patients with PCNSL compared with the glioblastoma group. miRNA, microRNA; PCNSL, primary central nervous system lymphoma; ROC, receiver operating characteristic; AUC, area under curve; CI, confidence interval.

high dose MTX-based chemotherapy. The treatment regimen consisted of chemotherapy alone or followed by whole brain radiation therapy. The effects of treatment were evaluated following three cycles of chemotherapy. The complete remission rate was $52 \%$, partial remission was $36 \%$, stability was $8 \%$ and disease progression was $4 \%$. The total response rate was $88 \%$. miRNA-21 was detected in patients with PCNSL prior to, following one cycle, and following three cycles of chemotherapy. miRNA-21 in patients with PCNSL had a relatively high expression level of $15.12 \pm 1.98$ prior to and $14.23 \pm 1.81$ following one cycle of chemotherapy $(\mathrm{P}>0.05)$, and as low as $2.94 \pm 1.15$ following 3 courses of chemotherapy (of the 22 cases that responded; $\mathrm{P}<0.001$, Fig. 4). However, there was no significant difference between the expression levels of miRNA-21 in patients prior to treatment compared with those in patients following one course of chemotherapy. The CSF miRNA-21 value prior to treatment minus the value following chemotherapy was calculated. Variations in CSF miRNA-21 were much higher prior to and following chemotherapy in patients who responded to chemotherapy compared with those in cases who had no response ( $\mathrm{P}=0.006$; Fig. 5). Spearman's rank correlation coefficient confirmed the reliability and validity of this biomarker (Spearman's rho $=0.563, \mathrm{P}=0.003$; data not shown).

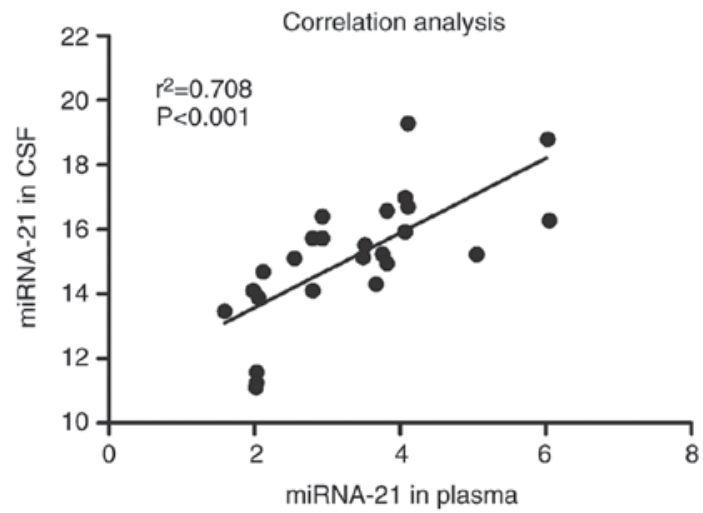

Figure 3. Pearson's correlation analysis of miRNA-21 in paired plasma and CSF. The expression of miRNA-21 in CSF and plasma was positively correlated. Pearson's correlation coefficient: $\mathrm{r}^{2}=0.708, \mathrm{P}<0.001$. miRNA, microRNA; CSF, cerebrospinal fluid.

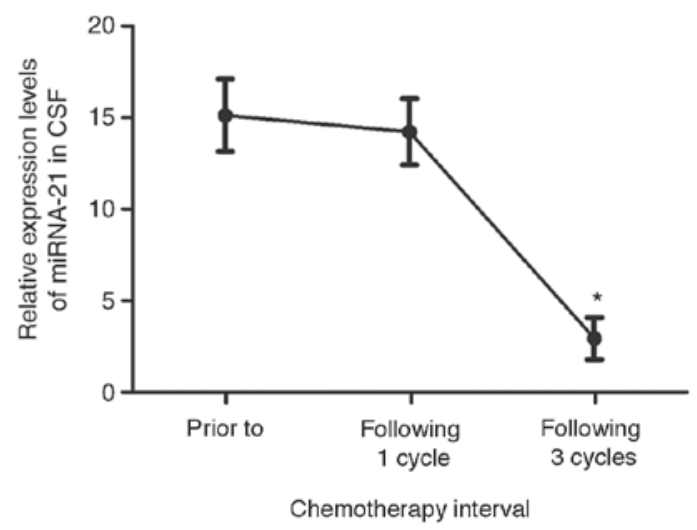

Figure 4. Expression levels of miRNA-21 in the CSF of patients with PCNSL, prior to and following chemotherapy. The relative expression of miRNA-21 was high prior to chemotherapy, with a downward tendency following one cycle of chemotherapy, but the results were not statistically significant. miRNA-21 expression decreased significantly following three cycles of chemotherapy in patients who responded to treatment. Results are displayed as the mean \pm standard deviation. ${ }^{*} \mathrm{P}<0.001$ vs. prior to chemotherapy. miRNA, microRNA; PCNSL, primary central nervous system lymphoma; CSF, cerebrospinal fluid.

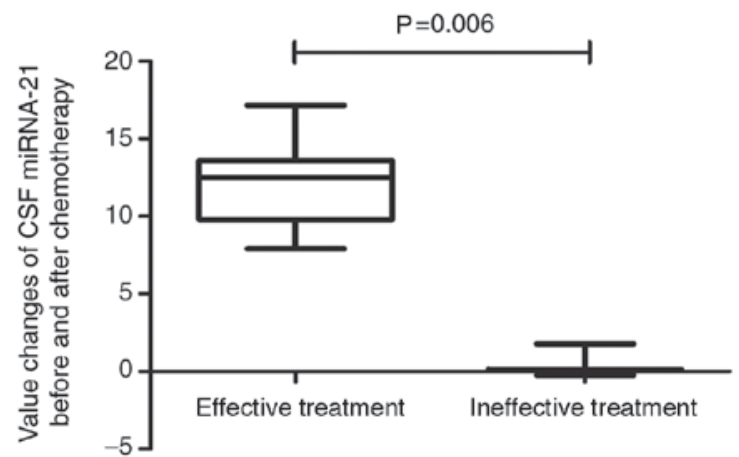

Figure 5. Expression levels of miRNA-21 in the CSF prior to and following chemotherapy. Results are in patients with PCNSL for whom chemotherapy was effective, compared with those for whom treatment was ineffective. CSF miRNA-21 values prior to treatment minus the value following chemotherapy were calculated. The range of CSF miRNA-21 expression levels was greater prior to and following chemotherapy in patients who responded to treatment, compared with those in cases who had no response $(\mathrm{P}=0.006)$. Results are displayed as the mean \pm standard deviation. miRNA, microRNA; PCNSL, primary central nervous system lymphoma; CSF, cerebrospinal fluid. 


\section{Discussion}

PCNSL is a type of non-Hodgkin's lymphoma with a high proliferative tumor index of $>50 \%$. However, the incidence of PCNSL in the general population is low, and accounts for $\sim 3 \%$ of all CNS tumors (1). Patients' specimens are difficult to collect; in the present study, plasma and CSF were collected from 25 patients with PCNSL, with an average age of 56.6 years, and $92 \%$ of PCNSL cases were positive for DLBCL. This is consistent with earlier published data indicating that the median age of onset for PCNSL in patients without other potential immune diseases is between 50 and 65 years (11). Owing to the involvement of the immune response, T cells and macrophages are frequently present in PCNSL lesions. Long-term immunosuppressive therapy or underlying disease is a considerable risk factor for PCNSL. PCNSL is more common in patients suffering from chronic inflammatory diseases, including systemic lupus erythematosus, tuberculosis, vasculitis, serious congenital immunodeficiency, AIDS, or those who have undergone immunosuppressive treatment as a result of transplantation (2). However, the discovery of the etiology of nervous system symptoms and brain lesions in patients with PCNSL remains a challenge. Immunosuppression is also the cause of other common, lethal brain tumors including glioblastoma, and it is difficult to distinguish between PCNSL and glioblastoma with imaging methods alone (12). In patients with suspected PCNSL, brain tumor stereotactic biopsy is the most effective diagnostic method. However, CNS biopsy carries a risk of bleeding and brain tissue damage. Therefore, an improved diagnostic approach is required.

The symptoms of PCNSL are also observable in other CNS disorders. In the majority of cases of DLBCL lymphoma with poor outcome, LDH is elevated (13). However, in the present study, LDH was normal in $76 \%$ of PCNSL cases. In the majority of cases, routine CSF assessment, leukocyte count and sugar content were within the normal limits, as was the protein content in $32 \%$ of cases. As a number of the patients displayed no obvious signs of disease, PCNSL cannot be diagnosed by the routine examination of CSF and biochemistry.

There is growing evidence to suggest that an imbalance in miRNA expression is evident in various types of cancer, and that miRNAs may act as oncogenes and tumor suppressor genes $(5,14)$. Alterations in miRNA expression levels are apparent in various malignant tumors, including leukemia and lymphoma, thus miRNAs are increasingly used as diagnostic and prognostic markers (15-17). The direct extraction of miRNAs from tumor samples (18) and simple detection of circulating miRNAs in human serum or plasma is also advantageous. Furthermore, it has been reported that miRNAs predict the clinical course of malignant tumors, including chronic lymphocytic leukemia and acute myeloid leukemia, in addition to certain organic diseases, including pancreatic tumors $(19,20)$. Therefore, a miRNA biomarker may be useful in the detection of PCNSL.

It has also been reported that the CSF expression levels of miRNA-21, miRNA-19, and miRNA-92a are increased in patients with PCNSL (21). Baraniskin et al (22) detected miRNA-15b and miRNA-21 expression levels in CSF through a comparative study of 23 patients with PCNSL, 10 patients with glioblastoma, 7 patients with brain metastases, and 10 control patients with various neurological disorders. The inclusion of miRNA-15b and miRNA-21 in combined expression analyses resulted in a diagnostic accuracy of $90 \%$ sensitivity and $100 \%$ specificity to distinguish patients with glioblastoma from those with PCNSL. Baraniskin et al (23) later confirmed their previous findings in an enlarged PCNSL cohort $(n=39$; sensitivity 97.4\%). In combined analyses of miRNA-21, miRNA-19b, and miRNA-92 in CSF, it was possible to differentiate PCNSL from other neurological disorders.

There is a certain degree of miRNA-21 expression in normal human plasma, where it is involved in the regulation of cell growth and differentiation (14). Therefore, miRNA-21 may contribute to the development and progression of disease, though only a small fraction of the biological functions of miRNA have been elucidated. Conti et al (24) and Wei et al (25) demonstrated that the expression level of miRNA-21 increased 7-11-fold in patients with glioblastoma compared with the normal control group, suggesting that miRNA-21 may be used for tumor diagnosis and staging.

Although obtaining CSF through a lumbar puncture is less invasive compared with a brain tumor biopsy, blood examination is a yet more favorable option. Lawrie et al (26) were able to detect miRNAs in the serum of patients with DLBCL. However, Baraniskin et al (23) revealed that miRNA levels in the serum of patients with PCNSL $(n=14)$ were not elevated compared with those of the control groups, including those with various neurological disorders (headache, seizures, syncope and stroke); this suggested that the blood-brain barrier was responsible for the differences between CSF and blood circulation in patients with PCNSL, and the small sample size may have been a further hindrance. In the present study, Pearson's correlation analysis revealed that miRNA-21 expression levels in the plasma were significantly positively correlated with those in the CSF. These results and those of a previous study (27) confirmed that it was possible to detect miRNA-21 in the plasma, as opposed to the CSF, of patients with PCNSL; Mao et al (27) revealed that expression levels of serum miRNA-21 did not differ significantly among CNS inflammation, metastases and healthy control groups. Thus, this was not repeated in the present study, and only glioblastoma (another common CNS tumor), and the healthy control groups were designated. RT-qPCR was used to confirm that miRNA-21 expression levels were higher in the plasma of patients with PCNSL, compared with those in the glioblastoma and healthy control groups. miRNA-21 expression in the plasma of patients with glioblastoma was also significantly higher compared with those in the control group. These results advocate plasma miRNA-21 to be a biomarker for the diagnosis of glioblastoma and PCNSL; lower miRNA-21 expression levels suggest glioblastoma, and higher levels suggest PCNSL. The results were consistent with an aforementioned previous study (28), in which the authors proposed that serum miRNA-21 expression levels were of value in identifying and prognostically stratifying patients with PCNSL, though they did not conclude whether miRNA-21 may be used to monitor the efficacy of chemotherapy.

Studies have demonstrated that aberrantly expressed miRNAs are associated with disease stage, drug resistance and survival in a large proportion of patients with cancer. Therefore, targeting these specific miRNAs may provide an efficient and optimal approach to the treatment of these types of cancer (29-32). 
The study by Baraniskin et al (23) provided evidence that CSF miRNAs have great potential as biomarkers for monitoring the treatment and disease follow-up of patients with PCNSL.

In the present study, patients were predominantly treated with high dose MTX-based chemotherapy combined with intrathecal injection, and only one patient with paraplegia was first administered radiotherapy $(6,28,33)$; these patients had a high response rate to treatment (88\%). To investigate the variation in miRNA-21 expression level in the CSF, miRNA-21 was subsequently detected prior to and following chemotherapy. Although the level of miRNA-21 expression decreased following one course of chemotherapy, there was no significant difference prior to and following treatment. However, the miRNA-21 expression level decreased significantly following three cycles of chemotherapy in patients responsive to treatment, while there were no obvious alterations in expression level in patients who did not respond to treatment $(\mathrm{n}=3)$. These results indicated that certain chemotherapeutic agents may exhibit anti-cancerous activity through the regulation of miRNA expression, which may influence cellular processes including DNA repair, cell cycle arrest, and apoptosis (34). This also suggests that miRNA-21 in the CSF may serve an important role in the evaluation of therapeutic effect and prognostic assessment in PCNSL.

In brief, miRNA-21 used as a diagnostic and therapeutic evaluation biomarker of PCNSL, is not only safe and convenient, but may also improve the accuracy of PCNSL diagnosis and prognostic estimation. The use of miRNA-21 as a tumor marker may also have potential in the clinical diagnosis and treatment of PCNSL.

\section{Acknowledgements}

The authors would like to thank Professor Li Ying for her guidance and help with the experiment.

\section{Funding}

The present study was supported by the National Key Basic Research Development Plan Corpus (grant no. 2012CBA01303), Harbin Medical University Scientific Research Innovation Projects (grant no. 2016LCZX61), and Health and Family Planning Commission Research Project in Heilongjiang province (grant no. 2016-018).

\section{Availability of data and materials}

The datasets used or analyzed during the current study are available from the corresponding author on reasonable request.

\section{Authors' contributions}

SW designed the study and analyzed the data. KY wrote the manuscript and analyzed the data. YC, YT and JH performed the laboratory work. YC and YT also analyzed the data. All authors read and approved the final manuscript.

\section{Ethics approval and consent to participate}

The present study was approved by the Harbin Medical University (Harbin, China) Ethics Committee, and all blood samples or cerebrospinal fluid (CSF) samples were used with the written informed consent of the patients.

\section{Patient consent for publication}

Both patients and volunteers provided written informed consent for the publication of this study.

\section{Competing interests}

The authors declare that they have no competing interests.

\section{References}

1. Dahiya S, Murphy ES, Chao ST, Stevens GH, Peereboom DM and Ahluwalia MS: Recurrent or refractory primary central nervous lymphoma: Therapeutic considerations. Expert Rev Anticancer Ther 13: 1109-1119, 2013.

2. Kleinschmidt-DeMasters BK, Damek DM, Lillehei KO, Dogan A and Giannini C: Epstein Barr virus-associated primary CNS lymphomas in elderly patients on immunosuppressive medications. J Neuropathol Exp Neurol 67: 1103-1111, 2008.

3. Löw S and Batchelor TT: Primary central nervous system lymphoma. Semin Neurol 38: 86-94, 2018.

4. Zorofchian S, El-Achi H, Yan Y, Esquenazi Y and Ballester LY: Characterization of genomic alterations in primary central nervous system lymphomas. J Neurooncol 140: 509-517, 2018.

5. Yu X, Li Z, Shen J, Chan MT and Wu WK: Role of microRNAs in primary central nervous system lymphomas. Cell Prolif 49: 147-153, 2016.

6. Royer-Perron L, Hoang-Xuan K and Alentorn A: Primary central nervous system lymphoma: Time for diagnostic biomarkers and biotherapies? Curr Opin Neurol 30: 669-676, 2017.

7. Zheng J, Xu J, Ma S, Sun X, Geng $M$ and Wang L: Clinicopathological study of gene rearrangement and microRNA expression of primary central nervous system diffuse large B-cell lymphomas. Int J Clin Exp Pathol 6: 2048-2055, 2013.

8. Musilova K and Mraz M: MicroRNAs in B-cell lymphomas: How a complex biology gets more complex. Leukemia 29: 1004-1017, 2015.

9. Crooks V, Waller S, Smith T and Hahn TJ: The use of the karnofsky performance scale in determining outcomes and risk in geriatric outpatients. J Gerontol 46: M139-M144, 1991.

10. Livak KJ and Schmittgen TD: Analysis of relative gene expression data using real-time quantitative PCR and the 2(-Delta Delta C(T)) method. Methods 25: 402-408, 2001.

11. Villano JL, Koshy M, Shaikh H, Dolecek TA and McCarthy BJ: Age, gender, and racial differences in incidence and survival in primary CNS lymphoma. Br J Cancer 105: 1414-1418, 2011.

12. Wu X, Nerisho S, Dastidar P, Ryymin P, Järvenpää R, Pertovaara H, Eskola $\mathrm{H}$ and Kellokumpu-Lehtinen PL: Comparison of different MRI sequences in lesion detection and early response evaluation of diffuse large B-cell lymphoma - a whole-body MRI and diffusion-weighted imaging study. NMR Biomed 26: 1186-1194, 2013.

13. Ichiki A, Carreras J, Miyaoka M, Kikuti YY, Jibiki T, Tazume K, Watanabe S, Sasao T, Obayashi Y, Onizuka M, et al: Clinicopathological analysis of 320 cases of diffuse large B-cell lymphoma using the hans classifier. J Clin Exp Hematop 57: 54-63, 2017.

14. Wu W, Sun M, Zou GM and Chen J: MicroRNA and cancer: Current status and prospective. Int J Cancer 120: 953-960, 2007.

15. Wang Z, Han J, Cui Y, Fan K and Zhou X: Circulating microRNA-21 as noninvasive predictive biomarker for response in cancer immunotherapy. Med Hypotheses 81: 41-43, 2013.

16. Ivo D'Urso P, Fernando D'Urso O, Damiano Gianfreda C, Mezzolla V, Storelli C and Marsigliante S: miR-15b and miR-21 as circulating biomarkers for diagnosis of glioma. Curr Genomics 16: 304-311, 2015.

17. Roth P, Keller A, Hoheisel JD, Codo P, Bauer AS, Backes C, Leidinger P, Meese E, Thiel E, Korfel A and Weller M: Differentially regulated miRNAs as prognostic biomarkers in the blood of primary CNS lymphoma patients. Eur J Cancer 51: 382-390, 2015. 
18. Calin GA, Sevignani C, Dumitru CD, Hyslop T, Noch E, Yendamuri S, Shimizu M, Rattan S, Bullrich F, Negrini M and Croce CM: Human microRNA genes are frequently located at fragile sites and genomic regions involved in cancers. Proc Natl Acad Sci USA 101: 2999-3004, 2004.

19. Gao HY, Wang W, Luo XG, Jiang YF, He X, Xu P, Chen X and Li XY: Screening of prognostic risk microRNAs for acute myeloid leukemia. Hematology 23: 747-755, 2018.

20. Greither T, Grochola LF, Udelnow A, Lautenschlager C, Würl P and Taubert H: Elevated expression of microRNAs 155, 203, 210 and 222 in pancreatic tumors is associated with poorer survival. Int J Cancer 126: 73-80, 2010.

21. Baraniskin A, Kuhnhenn J, Schlegel U, Chan A, Deckert M, Gold R, Maghnouj A, Zöllner H, Reinacher-Schick A, Schmiegel W, et al: Identification of microRNAs in the cerebrospinal fluid as marker for primary diffuse large B-cell lymphoma of the central nervous system. Blood 117: 3140-3146, 2011.

22. Baraniskin A, Kuhnhenn J, Schlegel U, Maghnouj A, Zöllner H, Schmiegel W, Hahn S and Schroers R: Identification of microRNAs in the cerebrospinal fluid as biomarker for the diagnosis of glioma. Neuro Oncol 14: 29-33, 2012.

23. Baraniskin A, Kuhnhenn J, Schlegel U, Schmiegel W, Hahn S and Schroers R: MicroRNAs in cerebrospinal fluid as biomarker for disease course monitoring in primary central nervous system lymphoma. J Neurooncol 109: 239-244, 2012.

24. Conti A, Aguennouz M, La Torre D, Tomasello C, Cardali S, Angileri FF, Maio F, Cama A, Germanò A, Vita G and Tomasello F: miR-21 and 221 upregulation and miR-181b downregulation in human grade II-IV astrocytic tumors. J Neurooncol 93: 325-332, 2009.

25. Wei D, Wan Q, Li L, Jin H, Liu Y, Wang Y and Zhang G: MicroRNAs as potential biomarkers for diagnosing cancers of central nervous system: A meta-analysis. Mol Neurobiol 51: $1452-1461,2015$

26. Lawrie CH,GalS,DunlopHM,PushkaranB,Liggins AP,Pulford K Banham AH, Pezzella F, Boultwood J, Wainscoat JS, et al: Detection of elevated levels of tumour-associated microRNAs in serum of patients with diffuse large B-cell lymphoma. Br J Haematol 141: 672-675, 2008.
27. Mao X, Sun Y and Tang J: Serum miR-21 is a diagnostic and prognostic marker of primary central nervous system lymphoma. Neurol Sci 35: 233-238, 2014

28. Rubenstein JL, Gupta NK, Mannis GN, Lamarre AK and Treseler P: How I treat CNS lymphomas. Blood 122: 2318-2330, 2013.

29. Li Y and Sarkar FH: MicroRNA targeted therapeutic approach for pancreatic cancer. Int J Biol Sci 12: 326-337, 2016.

30. Gasparini P, Cascione L, Landi L, Carasi S, Lovat F, Tibaldi C, Alì G, D'Incecco A, Minuti G, Chella A, et al: microRNA classifiers are powerful diagnostic/prognostic tools in ALK-, EGFR-, and KRAS-driven lung cancers. Proc Natl Acad Sci USA 112: 14924-14929, 2015.

31. Winther M, Alsner J, Tramm T, Baeksgaard L, Holtved E and Nordsmark M: Evaluation of miR-21 and miR-375 as prognostic biomarkers in esophageal cancer. Acta Oncol 54: 1582-1591, 2015.

32. Koutova L, Sterbova M, Pazourkova E, Pospisilova S, Svobodova I, Horinek A, Lysak D and Korabecna M: The impact of standard chemotherapy on miRNA signature in plasma in AML patients. Leuk Res 39: 1389-1395, 2015.

33. Wang CC, Carnevale J and Rubenstein JL: Progress in central nervous system Lymphomas. Br J Haematol 166: 311-325, 2014.

34. Chakraborty C, Doss CG, Sarin R, Hsu MJ and Agoramoorthy G: Can the chemotherapeutic agents perform anticancer activity through miRNA expression regulation? Proposing a new hypothesis [corrected]. Protoplasma 252: 1603-1610, 2015. 